\title{
Prospects for stochastic background searches using Virgo and LSC interferometers
}

\author{
Giancarlo Cella $^{1}$, Carlo Nicola Colacino ${ }^{2}$, Elena Cuoco ${ }^{3}$, \\ Angela Di Virgilio ${ }^{1}$, Tania Regimbau ${ }^{4}$, Emma L Robinson ${ }^{2}$ and \\ John T Whelan ${ }^{5}$ (for the LSC-Virgo working group on stochastic \\ backgrounds) \\ ${ }^{1}$ Istituto Nazionale di Fisica Nucleare sez. Pisa, 56100 Pisa, Italy \\ ${ }^{2}$ University of Birmingham, Edgbaston, Birmingham, B15 2TT, UK \\ 3 European Gravitational Observatory, 56021 Cascina (PI), Italy \\ ${ }^{4}$ Dpt. ARTEMIS, Observatoire de la Côte d'Azur, BP 42906304 Nice, France \\ 5 Max-Planck-Institut für Gravitationsphysik (Albert-Einstein-Institut), D-14476 Potsdam, \\ Germany \\ E-mail: john.whelan@aei.mpg.de
}

Received 23 April 2007, in final form 9 July 2007

Published 19 September 2007

Online at stacks.iop.org/CQG/24/S639

\begin{abstract}
We consider the question of cross-correlation measurements using Virgo and the LSC Interferometers (LIGO Livingston, LIGO Hanford and GEO600) to search for a stochastic gravitational-wave background. We find that inclusion of Virgo into the network will substantially improve the sensitivity to correlations above $200 \mathrm{~Hz}$ if all detectors are operating at their design sensitivity. This is illustrated using a simulated isotropic stochastic background signal, generated with an astrophysically-motivated spectral shape, injected into $24 \mathrm{~h}$ of simulated noise for the LIGO and Virgo interferometers.
\end{abstract}

PACS numbers: $04.80 . \mathrm{Nn}$, 04.30.Db, 07.05.Kf, 95.55.Ym

\section{Introduction}

There are four kilometre-scale interferometric gravitational-wave (GW) detectors currently in operation: the $4 \mathrm{~km}$ and $2 \mathrm{~km}$ interferometers (IFOs) at the LIGO Hanford Observatory (LHO) (known respectively as $\mathrm{H} 1$ and $\mathrm{H} 2$ ), the $4 \mathrm{~km}$ IFO at the LIGO Livingston Observatory (LLO) (known as L1) and the $3 \mathrm{~km}$ Virgo IFO (known as V1). The LLO and LHO IFOs are currently conducting operations at their design sensitivity, and operate together with the $600 \mathrm{~m}$ GEO600 IFO (known as G1) under the auspices of the LIGO Scientific Collaboration (LSC). Virgo's first full science run commences in May 2007.

One of the signals targeted by ground-based GW IFOs is a stochastic GW background (SGWB), which can either be of cosmological or astrophysical origin, in the latter case being 
produced by a superposition of unresolved sources. The standard technique to search for an SGWB looks for correlations in the outputs of multiple detectors. We describe in this paper how the inclusion of correlation measurements involving Virgo could improve the sensitivity of the current LLO-LHO network.

\section{All-sky sensitivity at design}

\subsection{Observing geometry}

The effect of a SGWB is to generate correlations in the outputs of a pair of GW detectors, which can be described for an isotropic background in the Fourier domain by

$$
\left\langle\widetilde{h}_{1}^{*}(f) \widetilde{h}_{2}\left(f^{\prime}\right)\right\rangle=\frac{1}{2} \delta\left(f-f^{\prime}\right) \gamma_{12}(f) S_{\mathrm{gw}}(f) .
$$

The raw correlation depends on the (one-sided) power spectral density $S_{\mathrm{gw}}(f)$ the SGWB would generate in an IFO with perpendicular arms, as well as the observing geometry. The geometrical dependence manifests itself via the overlap reduction function (ORF) [1], which can be written as [2]

$$
\gamma_{12}(f)=d_{1 a b} d_{2}^{c d} \frac{5}{4 \pi} \iint \mathrm{d}^{2} \Omega_{\hat{n}} P_{c d}^{\mathrm{TT} \hat{a} a b} \mathrm{e}^{\mathrm{i} 2 \pi f \hat{n} \cdot\left(\vec{r}_{2}-\vec{r}_{1}\right) / c}
$$

where each IFO's geometry is described by a response tensor constructed from unit vectors $\hat{x}$ and $\hat{y}$ down the two arms

$$
d^{a b}=\frac{1}{2}\left(\hat{x}^{a} \hat{x}^{b}-\hat{y}^{a} \hat{y}^{b}\right),
$$

$\vec{r}_{1,2}$ is the respective interferometer's location and $P_{c d}^{\text {TTñab }}$ is a projector onto traceless symmetric tensors transverse to the unit vector $\hat{n}$. At zero frequency, the ORF is determined entirely by detector orientation. The LHO and LLO sites are aligned as nearly as possible given their separation on the globe, so that $\gamma_{\mathrm{HL}}(0)=-0.89$. In contrast, the Virgo and GEO600 sites are poorly oriented with respect to one another, so $\gamma_{\mathrm{GV}}(0)=-0.08$. However, the frequency-dependence of the ORFs means that the situation is quite different at frequencies above $40 \mathrm{~Hz}$, where the IFOs are sensitive. In particular, the amplitude of $\gamma_{\mathrm{GV}}(f)$ does not drop appreciably for $f$ below about $350 \mathrm{~Hz}$. For the other pairs, the behaviour is determined by the high-frequency limiting form of the ORF,

$$
\gamma_{12}(f) \longrightarrow 5 d_{1 a b} P_{c d}^{\mathrm{TT}_{\hat{s}_{12} a b}} d_{2}^{c d} \operatorname{sinc}\left(2 \pi f T_{12}\right)=\frac{\gamma_{12}^{\mathrm{env}}}{f} \sin \left(2 \pi f T_{12}\right)
$$

where $T_{12}$ is the light travel time between the detector sites and $\hat{s}_{12}$ is a unit vector pointing from one site to the other. While trans-Atlantic light travel times like $T_{\mathrm{HV}}$ and $T_{\mathrm{LV}}$ are greater than $T_{\mathrm{HL}}$, leading to more oscillations in the ORF, the overall envelope $\gamma_{12}^{\text {env }}=\left(5 d_{1 a b} P_{c d}^{\mathrm{TT}_{\hat{s}_{12}} a b} d_{2}^{c d}\right) /\left(2 \pi T_{12}\right)$ includes geometric projection factors, which more than make up for this discrepancy, as summarized in table 1 . The result is that in the full overlap reduction function (figure 1) the typical amplitudes $\left|\gamma_{\mathrm{HV}}(f)\right|$ and $\left|\gamma_{\mathrm{LV}}(f)\right|$ are larger than the typical $\left|\gamma_{\mathrm{HL}}(f)\right|$ for $f \gtrsim 200 \mathrm{~Hz}$.

\subsection{Definition of sensitivity}

The strength of an isotropic stochastic background can be written in terms of the one-sided power spectral density $S_{\mathrm{gw}}(f)$ it would generate in an IFO with perpendicular arms.

The standard cross-correlation method seeks to measure the amplitude $S_{R}=$ $S_{\mathrm{gw}}(f) / \mathcal{S}(f)$ of a background whose $S_{\mathrm{gw}}(f)$ is assumed to have a specified shape $\mathcal{S}(f)$. 


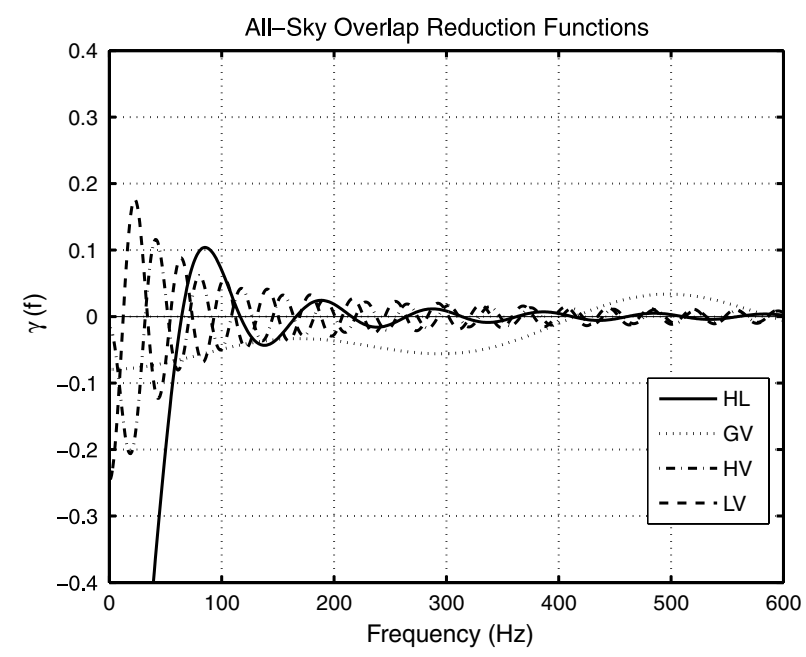

Figure 1. The overlap reduction functions for pairs of detector sites. Note that the ORF for the two LIGO sites goes off the scale of this plot at $40 \mathrm{~Hz}$, which is the 'seismic wall' below which LIGO data are too noisy to be of any use. The proximity and alignment of LLO and LHO makes HL the most favourable pair of detector sites for observations below $150 \mathrm{~Hz}$ or so. However, the proximity of the Virgo and GEO600 sites means the GV ORF is substantial out to higher frequencies, overcoming the low-frequency suppression due to their poor alignment. On the other hand, as shown in table 1 the HV and LV ORFs, while they oscillate rapidly with increasing frequency, do not decay as precipitously as the HL ORF, making them more favourable than HL (but less than GV) for $f \gtrsim 200 \mathrm{~Hz}$.

Table 1. Limiting behaviour of overlap reduction functions of detector pairs. H refers to either of the IFOs at the LHO site, L to LLO, V to Virgo and G to GEO600. At $f=0$, the ORF is determined by the alignment of the detectors. The reduced inverse light travel time $\left(2 \pi T_{12}\right)^{-1}$ gives a characteristic frequency for the onset of 'high-frequency' behaviour, which includes a sinc function of the ratio of $f$ to that frequency. However, the limiting form in (4) also includes a geometric projection factor, leading to an overall envelope $\gamma_{12}^{\text {env }}$ which is shown in the third row. In particular, while the light-travel time $T_{\mathrm{HL}}$ is less than $T_{\mathrm{HV}}$ or $T_{\mathrm{LV}}$, the projection factor more than makes up for this, which makes the mean amplitudes of $\gamma_{\mathrm{HV}}(f)$ and $\gamma_{\mathrm{LV}}(f)$ at high frequencies larger than that for $\gamma_{\mathrm{HL}}(f)$.

\begin{tabular}{lcccccc}
\hline & HL & HV & LV & GH & GL & GV \\
\hline$\gamma_{12}(0)$ & -0.89 & -0.02 & -0.25 & 0.42 & -0.32 & -0.08 \\
$\left(2 \pi T_{12}\right)^{-1}(\mathrm{~Hz})$ & 15.9 & 5.8 & 6.0 & 6.3 & 6.3 & 49.8 \\
$\gamma_{12}^{\text {env }}(\mathrm{Hz})$ & -1.7 & 5.0 & -5.9 & 3.0 & 4.3 & -17.2 \\
\hline
\end{tabular}

Given coincident data between times $t_{1}$ and $t_{2}$ from detectors with one-sided noise power spectral densities (PSDs) $P_{1,2}(f)$, we can make an optimally-filtered cross-correlation statistic

$$
Y=\int_{t_{1}}^{t_{2}} \int_{t_{1}}^{t_{2}} s_{1}(t) Q\left(t-t^{\prime}\right) s_{2}(t) \mathrm{d} t \mathrm{~d} t^{\prime}
$$

with the optimal filter defined by its Fourier transform

$$
\widetilde{Q}(f)=\mathcal{N} \frac{\gamma_{12}(f) \mathcal{S}(f)}{P_{1}(f) P_{2}(f)}
$$



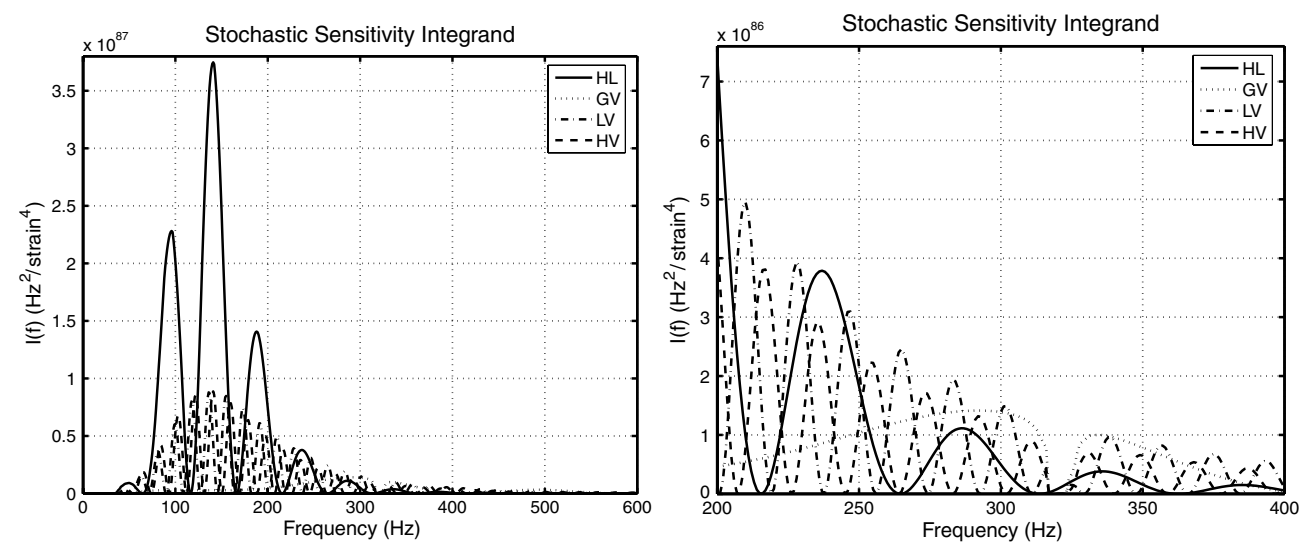

Figure 2. Sensitivity integrands $\mathcal{I}(f)$ for pairs of detector sites, where pairs like HL include, e.g., the combined sensitivity of H1-L1 and H2-L1. To get an overall sensitivity, these need to be multiplied by the square of the shape of the SGWB spectrum $S_{\mathrm{gw}}(f)$. The HL pair is the most sensitive for flat and low-frequency spectra, but as shown in the closeup at the right, above $200 \mathrm{~Hz}$, pairs involving Virgo are more sensitive when all detectors are operating at their design sensitivities. The GH and GL pairs are not shown, since the GEO600 noise spectrum [5] means that only the GV pair contributes significantly to the overall sensitivity at these frequencies.

and $\mathcal{N}$ chosen so that $\langle Y\rangle=S_{R}$. If the geometric mean of the noise PSDs is large compared to $S_{\mathrm{gw}}(f)$, the expected variance of the statistic will be

$$
\sigma^{2}=\frac{1}{2 T}\left(\int_{0}^{\infty} \frac{\left[\gamma_{12}(f) \mathcal{S}(f)\right]^{2}}{P_{1}(f) P_{2}(f)}\right)^{-1}
$$

where $T=t_{2}-t_{1}$ is the duration of the data analysed. The squared signal-to-noise ratio of the standard cross-correlation statistic will thus be

$$
\mathrm{SNR}^{2}:=\frac{\langle Y\rangle^{2}}{\sigma^{2}}=2 T S_{R}^{2} \int_{0}^{\infty}[\mathcal{S}(f)]^{2} \mathcal{I}_{12}(f) \mathrm{d} f
$$

where we have defined a 'sensitivity integrand' which illustrates the contribution to the sensitivity of different frequencies:

$$
\mathcal{I}_{12}(f)=\frac{\left[\gamma_{12}(f)\right]^{2}}{P_{1}(f) P_{2}(f)} .
$$

We plot $\mathcal{I}(f)$ for several pairs of detectors in figure 2, using the design sensitivities in [3-5]. As shown in [6], the optimal method for combining correlation measurements from different detector pairs is the same as that for combining measurements from different times: average the point estimates $Y$ with a relative weighting of $\sigma^{-2}$, and the resulting variance will be the inverse of the sum of the $\sigma^{-2}$ values. This produces a sensitivity integrand which is the sum of the integrands for individual pairs:

$$
\mathcal{I}(f)=\sum_{\text {pair }} \mathcal{I}_{\text {pair }}(f)
$$

An immediate application of this is to define sensitivity integrands that combine pairs involving $\mathrm{H} 1$ and $\mathrm{H} 2$, e.g., $\mathcal{I}_{\mathrm{HL}}=\mathcal{I}_{\mathrm{H} 1, \mathrm{~L} 1}+\mathcal{I}_{\mathrm{H} 2, \mathrm{~L} 1}$. This is the same as using the spectrum of an optimally combined $\mathrm{H}$ pseudo-detector as described in [7]. 

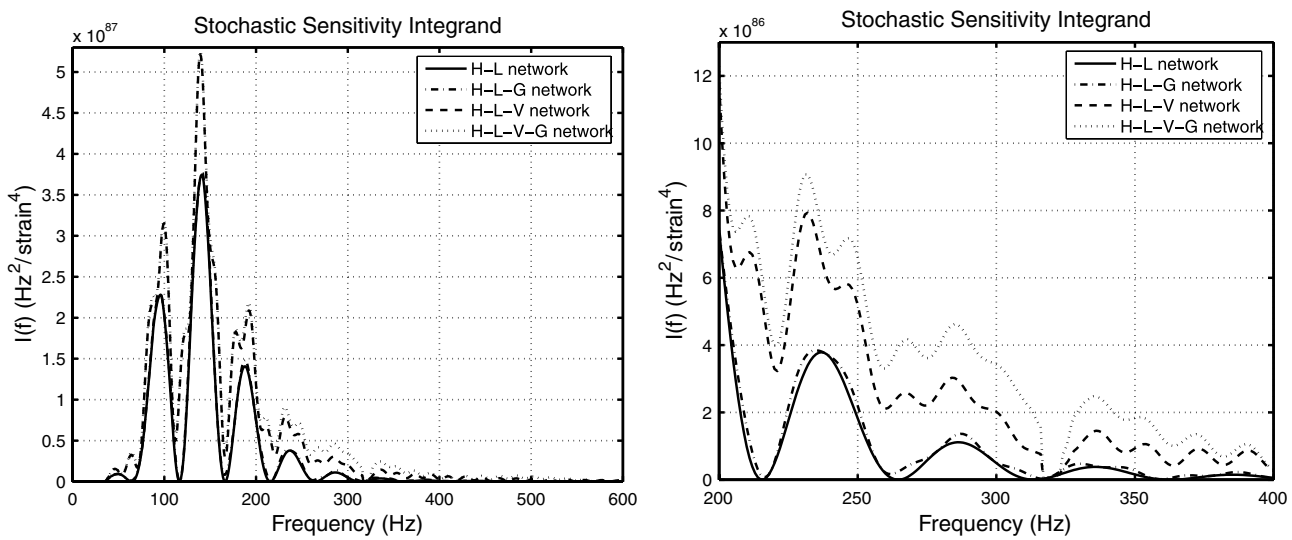

Figure 3. Combined sensitivity integrands for networks of detectors. In each case a network including ' $\mathrm{H}$ ' includes correlations between both $\mathrm{H} 1$ and $\mathrm{H} 2$ and the detectors at other sites. As the closeup on the right shows, addition of the LV, HV and GV pairs to the HL network increases sensitivity to backgrounds with significant power above $200 \mathrm{~Hz}$.

Table 2. Smallest detectable band-limited background using each of the detector networks defined in figure 3. In each case, this is the strain power spectrum, in units of $10^{-48} \mathrm{~Hz}^{-1}$, that could be detected with $5 \%$ false alarm and $5 \%$ false dismissal rates, using 1 year of coincident data at design sensitivity.

\begin{tabular}{lrrll}
\hline Band $(\mathrm{Hz})$ & H-L & H-L-G & H-L-V & H-L-V-G \\
\hline $200-300$ & 5.79 & 5.43 & 3.44 & 3.04 \\
$300-400$ & 18.57 & 15.37 & 7.92 & 5.88 \\
\hline
\end{tabular}

Figure 3 shows the combined sensitivity for four networks of detectors operating at design sensitivity: the existing $\mathrm{H}-\mathrm{L}$ network, an $\mathrm{H}-\mathrm{L}-\mathrm{G}$ network in which GEO600 is also operating at design sensitivity, and $\mathrm{H}-\mathrm{L}-\mathrm{V}$ and $\mathrm{H}-\mathrm{L}-\mathrm{V}-\mathrm{G}$ networks which also include a design-sensitivity Virgo. The H1-H2 pair is not included in these networks, because the presence of correlated environmental noise necessitates special treatment of this pair [8].

Since the power in the faintest detectable 'white' stochastic background is proportional to the square root of the area under the sensitivity integrand, we see that the addition of Virgo to the LHO-LLO network would be most useful in improving sensitivity to a narrowband background peaked above $200 \mathrm{~Hz}$, or to one whose spectrum rises with increasing frequency. As an illustration, table 2 shows for several detector networks the faintest detectable background with constant $S_{\mathrm{gw}}(f)$ in a $100 \mathrm{~Hz}$ band, assuming one year of observation time and an SNR threshold of 3.29, associated with 5\% false alarm and false dismissal rates.

\section{Simulations}

To test cross-correlation analyses of LIGO and Virgo data, we injected a simulated SGWB signal into simulated LIGO and Virgo noise. The simulated noise data were the $24 \mathrm{~h}$ of $\mathrm{H} 1, \mathrm{H} 2, \mathrm{~L} 1$ and V1 data, all at nominal design sensitivity, initially generated for simulated searches for GW bursts and inspiralling compact object binaries [9, 10], known as 'project $1 b$ '. We chose a spectral shape designed to highlight the performance of the LV and HV pairs 

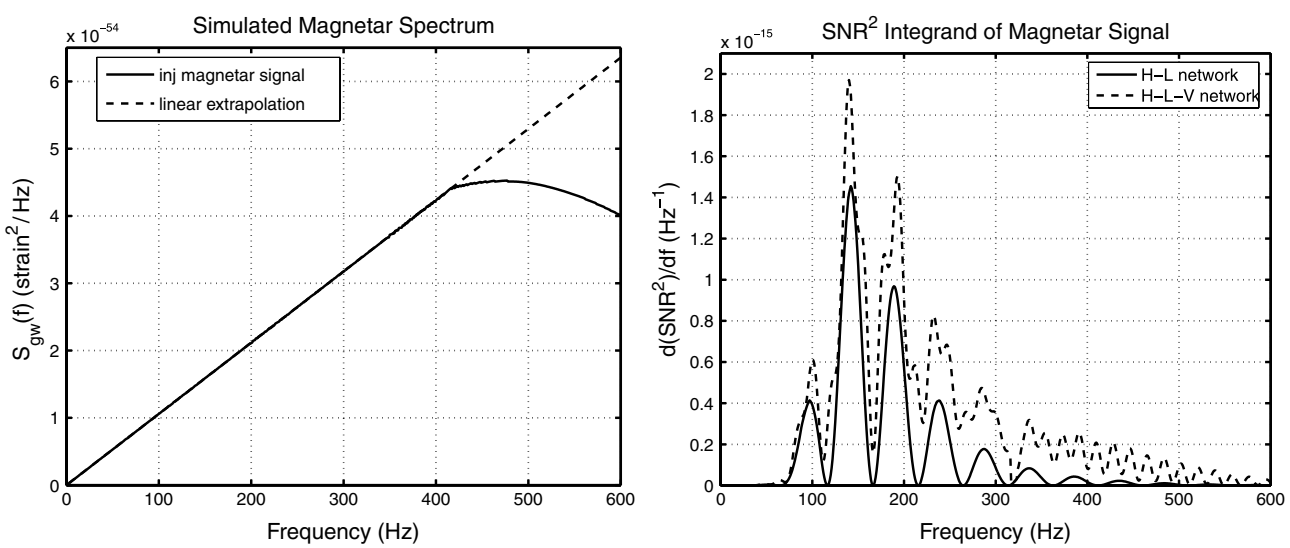

Figure 4. The magnetar spectrum used to generate simulated signals, and the associated integrand for squared signal-to-noise ratio. Note that since $S_{\mathrm{gw}}(f)$ increases approximately linearly with a frequency up to about $400 \mathrm{~Hz}$, this spectrum, while broad-band, tends to favour the higher frequencies where LIGO-Virgo detector pairs are more sensitive. Beyond $400 \mathrm{~Hz}$, the spectrum is no longer linear, but we show the SNR that would result from attempting to detect it with a $\mathcal{S}(f) \propto f$ filter; the corrections are negligible below about $500 \mathrm{~Hz}$, and still small throughout the frequency range displayed. Note that integrating the area under the $\mathrm{H}-\mathrm{L}-\mathrm{V}$ curve on the right still gives an SNR below $10^{-6}$ from $24 \mathrm{~h}$ of data, so we scale up the injected strain signal by a factor of several thousand in the simulations described in section 4 .

at $f \gtrsim 200 \mathrm{~Hz}$, but which corresponds to a model of an astrophysical SGWB. The spectrum we used is associated with the superposition of the tri-axial emission from the extra-galactic population of spinning magnetars with type I superconducting interior, as described in the model B of [11], but updated using the star formation history of [12].

In figure 4 we show the spectrum and the associated sensitivity integrand in the corresponding detectors. Since this spectrum rises with increasing frequency up to about $400 \mathrm{~Hz}$, it is useful for illustrating the utility of a network involving Virgo to search for a broad-band astrophysical source. Since the model signal would be too weak to detect with first-generation interferometers, we scale up the signal strength, injecting a signal with the same spectral shape, but a much larger amplitude, in our simulations.

\subsection{Simulation algorithm}

The problem of simulation of the signal in a pair of detectors due to an isotropic and Gaussian SGWB has been considered previously in e.g., $[6,13,14]$. For this work we generalize that to a network of $N \mathrm{GW}$ detectors ${ }^{6}$. We need to satisfy (1) for each pair of detectors; treating $\left\{h_{A}(f)\right\}$ as the elements of a column vector $\widetilde{\mathbf{h}}(f)$ and $\left\{\gamma_{A B}(f)\right\}$ as the elements of a real, symmetric matrix ${ }^{7} \gamma(f)$, we can write this as a matrix equation

$$
\left\langle\tilde{\mathbf{h}}(f) \tilde{\mathbf{h}}\left(f^{\prime}\right)^{\dagger}\right\rangle=\frac{1}{2} S_{\mathrm{gw}}(f) \gamma(f) \delta\left(f-f^{\prime}\right) .
$$

If we can define a matrix $\boldsymbol{\beta}(f)$ which factors $\gamma(f)$ :

$$
\gamma(f)=\boldsymbol{\beta}(f) \boldsymbol{\beta}(f)^{\dagger},
$$

6 In our case, to simulate signals in $\mathrm{H} 1, \mathrm{H} 2, \mathrm{~L} 1$ and $\mathrm{V} 1, N=3$, because $\mathrm{H} 1$ and $\mathrm{H} 2$ have the same response tensor and therefore the same simulated GW signal can be used for both of them.

7 For non-isotropic backgrounds, the ORF is complex rather than real, and more care must be taken with the definition of the Hermitian matrix $\gamma(f)$. 
then we can generate $N$-independent white noise data streams $\left\{\widetilde{\eta}_{A}(f)\right\}$ which satisfy

$$
\left\langle\widetilde{\boldsymbol{\eta}}(f) \widetilde{\boldsymbol{\eta}}\left(f^{\prime}\right)^{\dagger}\right\rangle=\mathbf{1} \delta\left(f-f^{\prime}\right)
$$

and then convert them into the desired coloured correlated data streams via

$$
\widetilde{\mathbf{h}}(f)=\sqrt{\frac{S_{\mathrm{gw}}(f)}{2}} \boldsymbol{\beta}(f) \widetilde{\boldsymbol{\eta}}(f) .
$$

For a given $\gamma(f)$, there are different choices of $\widetilde{\boldsymbol{\eta}}(f)$ which achieve the factorization (12).

Since (11) is a covariance matrix, it is positive semi-definite, from which it follows (since $S_{\mathrm{gw}}(f)>0$ ) that $\gamma(f)$ is positive semidefinite as well. $\gamma(f)$ could have one or more zero eigenvalues in the presence of linear dependence between detector outputs. A practical example of this is two detectors sharing the same geometry and location, such as $\mathrm{H} 1$ and $\mathrm{H} 2$, are included in the network ${ }^{8}$. We avoid this problem by generating a simulated signal for $\mathrm{H} 1$ and injecting it into both $\mathrm{H} 1$ and $\mathrm{H} 2$.

In the generic case where $\gamma(f)$ is positive definite, we can make the straightforward choice of the Cholesky decomposition [15], in which $\boldsymbol{\beta}(f)$ is a lower diagonal matrix. In the case $N=2$, this reduces to the form used in e.g., [6]. For $N=3$, if the diagonal elements of $\gamma(f)$ are unity ${ }^{9}$, the explicit form is

$$
\boldsymbol{\beta}(f)=\left(\begin{array}{ccc}
1 & 0 & 0 \\
\gamma_{12} & \sqrt{1-\gamma_{12}^{2}} & 0 \\
\gamma_{13} & \frac{\gamma_{23}-\gamma_{12} \gamma_{13}}{\sqrt{1-\gamma_{12}^{2}}} & \sqrt{\frac{1+2 \gamma_{12} \gamma_{13} \gamma_{23}-\gamma_{12}^{2}-\gamma_{13}^{2}-\gamma_{23}^{2}}{1-\gamma_{12}^{2}}}
\end{array}\right) .
$$

However in practice we can simply use a fast iterative algorithm for the Cholesky decomposition.

Other factorization strategies which treat the different detectors more symmetrically (e.g., defining $\boldsymbol{\beta}(f)=\boldsymbol{\Lambda}(f)^{1 / 2} \mathbf{U}(f)$ where $\boldsymbol{\Lambda}(f)$ is the diagonal matrix of eigenvalues of $\gamma(f)$ and $\mathbf{U}(f)$ is the matrix constructed from the corresponding eigenvectors) may be more demanding in terms of computational power, but more numerically stable when correlations between the detectors are large and off-diagonal elements of $\gamma(f)$ are comparable to unity. In particular, this strategy can deal directly with the case when $\gamma(f)$ has one or more zero eigenvalues.

\subsection{Filtering strategy}

The continuous frequency-domain idealization (14) needs to be applied with care to finite stretches of real detector data. In the time domain, the multiplication (14) amounts to a convolution

$$
\mathbf{h}(t)=\int_{-\infty}^{\infty} \mathbf{K}\left(t-t^{\prime}\right) \boldsymbol{\eta}\left(t^{\prime}\right) \mathrm{d} t^{\prime}
$$

with a kernel which is the inverse Fourier transform of

$$
\widetilde{\mathbf{K}}(f)=\sqrt{\frac{S_{\mathrm{gw}}(f)}{2}} \boldsymbol{\beta}(f) .
$$

If the time-domain kernel $\mathbf{K}(\tau)$ is negligible outside the interval $-\tau_{-}<\tau<\tau_{+}$, we can use the standard overlap-and-add strategy to generate a continuous stream of time-series data, as illustrated in figure 5.

8 Less trivial examples can be constructed, for example three detectors in the same location and in the same plane.

9 This is the case for interferometers with perpendicular arms, but not for GEO600 or for resonant bar detectors; see [2]. 


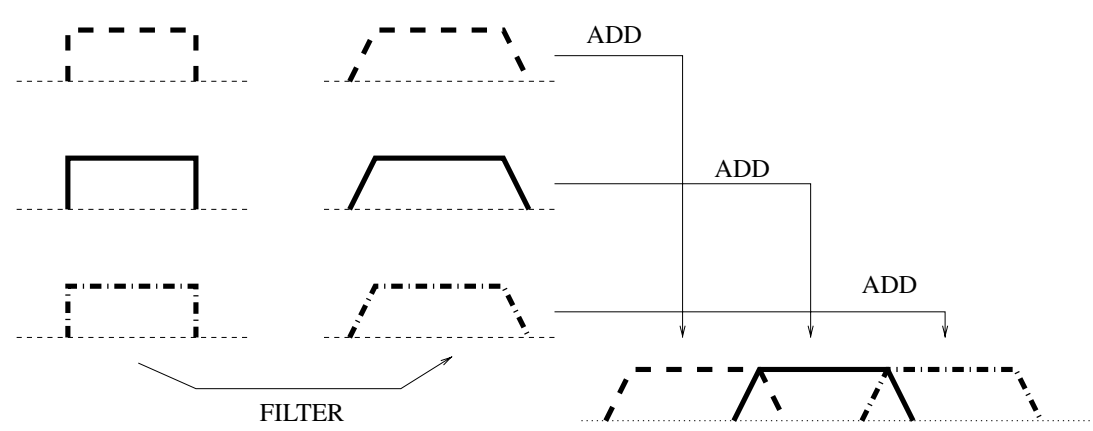

Figure 5. The overlap-and-add algorithm.

(i) Generate a sequence of 'buffers' of white noise data for each of the $N$ detectors, each of length $T$.

(ii) Convolve each buffer with the kernel $\mathbf{K}(\tau)$ to obtain a time series of length $\tau_{-}+T+\tau_{+}$, with an associated start time $\tau_{-}$before the start and end time $\tau_{+}$after the end of the original buffer. (This is most naturally done in the frequency domain, zero-padding the white noise by $\tau_{-}$at the beginning and $\tau_{+}$at the end, then Fourier transforming and multiplying by $\widetilde{\mathbf{K}}(f)$ before inverse-Fourier-transforming.)

(iii) Add together the processed data buffers, overlapping by $\tau_{+}$on one end and $\tau_{-}$on the next, producing correlated coloured time-series data of duration $T$ times the number of buffers, plus transients of $\tau_{-}$at the beginning and $\tau_{+}$at the end, which are discarded. [16].

This strategy was implemented in code based on Virgo's 'noise analysis package' (nap)

\section{Analysis of simulated data}

The continuous signals described in section 3 were injected into the 'project $1 \mathrm{~b}$ ' simulated noise and the resulting time series analysed using the matapps stochastic analysis code developed by the LSC [17]. In particular, the cross-correlation (5) was performed in the frequency domain without any need to resample the time-series data, using a variation of the method described in [18] and applied in [14]. Several simulation runs were performed in which the same set of simulated signals were injected into the four data streams, scaled up by a different factor for each run. The results of two of those simulations are shown here. In figure 6 we plot the individual point estimates and error bars in each of the five detector pairs (every combination except for $\mathrm{H} 1-\mathrm{H} 2$ ). The amplitude measure $S_{R}$ quoted is the one-sided strain PSD at $200 \mathrm{~Hz}$. The optimal filter used assumed the shape $\mathcal{S}(f) \propto f$, although the injected signals only had that form below about $400 \mathrm{~Hz}$. The analysis was done over a frequency band $50-500 \mathrm{~Hz}$ to avoid difficulties arising from this mismatch, as illustrated in figure 4.

The optimal combination of these results is shown in table 3 , both for the full network and for the network consisting only of the two LHO-LLO pairs. Our error bars are reduced by $15 \%$ via the inclusion of the LIGO-Virgo pairs. Note that although this source has more power at the intermediate frequencies favoured by the LIGO-Virgo pairs, it is still broad-band. A narrow-banded source with most of its power above $200 \mathrm{~Hz}$ would favour LIGO-Virgo pairs to an even greater extent. 


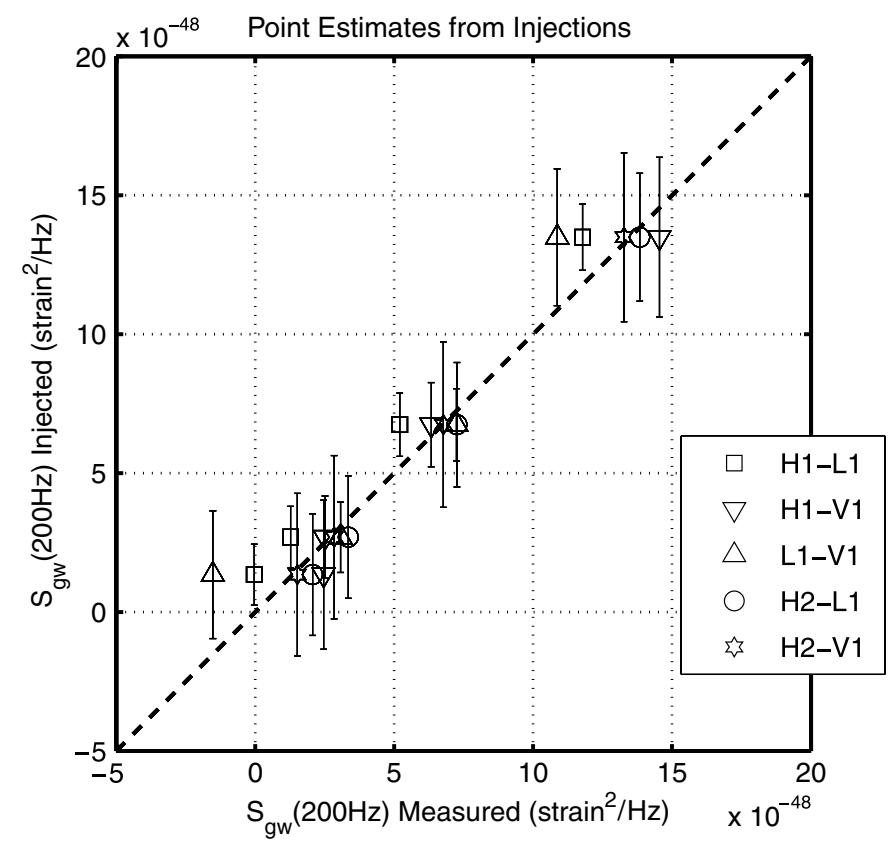

Figure 6. The individual point estimates and one-sigma error bars with signals injected at two different levels. The strength of the injection is shown on the vertical axis, as a strain power at $200 \mathrm{~Hz}$, while the estimate of this quantity from each detector pair is on the horizontal axis. The error bars are drawn vertically for ease of reading, and because they are frequentist error bars as quoted. Note that the signals are seen at comparable strength in all five pairs of detectors.

Table 3. The values of $S_{\mathrm{gw}}(200 \mathrm{~Hz})$ calculated from the simulated project $1 \mathrm{~b}$ data, with associated one-sigma error bars, for the H-L network consisting of the H1-L1 and H2-L1 pairs, and for the H-L-V network consisting of those plus the H1-V1, H2-V1 and L1-V1 pairs. We see that including Virgo in the network reduces our error bars for this broad-band astrophysical spectrum by $15 \%$.

\begin{tabular}{crr}
\hline \multicolumn{3}{c}{$S_{\mathrm{gw}}(200 \mathrm{~Hz})\left(10^{-48} \mathrm{~Hz}^{-1}\right)$} \\
\hline Injected & \multicolumn{1}{c}{ H-L Result } & \multicolumn{1}{c}{ H-L-V Result } \\
\hline 1.35 & $0.39 \pm 0.98$ & $0.43 \pm 0.82$ \\
2.70 & $1.69 \pm 0.99$ & $2.31 \pm 0.67$ \\
6.74 & $5.63 \pm 1.02$ & $6.29 \pm 0.69$ \\
13.49 & $12.22 \pm 1.06$ & $12.35 \pm 0.88$ \\
\hline
\end{tabular}

\section{Conclusions and outlook}

We have demonstrated how the inclusion of LIGO-Virgo and possibly GEO600-Virgo detector pairs can enhance the sensitivity of the global GW detector network to an isotropic background of gravitational waves, particularly at frequencies above $200 \mathrm{~Hz}$. As a practical illustration, we have adapted and applied pipelines for generating correlated simulated signals in the LSC and Virgo detectors, and for analysing coincident data via the standard cross-correlation technique. The specific astrophysical model we used (which was chosen because its frequency spectrum 
was peaked at frequencies where LIGO-Virgo pairs at design will be more sensitive than LLO-LHO pairs) had to have its amplitude increased to be detectable by any pair of firstgeneration IFOs. Nonetheless, the exercise illustrates how multiple detector pairs can be used to discover an 'unexpected' background.

Virgo is not yet at its nominal design sensitivity, but has improved its sensitivity markedly over the past year, and its first full science run starts in May 2007, to be analysed in conjunction with the end of LIGO's S5 run.

\section{Acknowledgments}

The authors would like to thank their colleagues in the LIGO Scientific Collaboration and the Virgo project. JTW gratefully acknowledges Loyola University New Orleans and the University of Texas at Brownsville. This work was supported by the National Science Foundation under grant PHY-0300609 and by the Max-Planck-Society. This paper has been assigned LIGO Document Number LIGO-P070028-03-Z and AEI document number AEI-2007-017.

\section{References}

[1] Flanagan É É 1993 Phys. Rev. D 482389 (Preprint astro-ph/9305029)

[2] Whelan J T 2006 Class. Quantum Grav. 231181 (Preprint gr-qc/0509109)

[3] Lazzarini A and Weiss R 1996 Technical Document LIGO-E950018-02-E Digital format from http://www.ligo. caltech.edu/ jzweizig/distribution/LSC_Data/

[4] Punturo M 2004 Virgo Note VIR-NOT-PER-1390-51 Issue 10, Digital format from http://www.virgo.infn.it/ senscurve/

[5] GEO-600 Theoretical Noise Budget version 4.0 (2006) with $250 \mathrm{~Hz}$ signal detuning, available from http://www.geo600.uni-hannover.de/geocurves/

[6] Allen B and Romano J D 1999 Phys. Rev. D 59102001 (Preprint gr-qc/9710117)

[7] Lazzarini A et al 2004 Phys. Rev. D 70062001 (Preprint gr-qc/0403093)

[8] Fotopoulos N V (for the LIGO Scientific Collaboration) 2006 Class. Quantum Grav. 23 S693

[9] Beauville F et al (LIGO-Virgo Working Group) 2007 Preprint gr-qc/0701026 (Class. Quantum Grav. submitted)

[10] Beauville F et al (LIGO-Virgo Working Group) 2007 Preprint gr-qc/0701027 (Class. Quantum Grav. submitted)

[11] Regimbau T and de Freitas Pacheco J A 2006 Astron. Astrophys. 4471

[12] Hopkins A M and Beacom J 2006 Astrophys. J. 651142 (Preprint astro-ph/0601463)

[13] Bose S et al 2003 Class. Quantum Grav. 20 S677

[14] Abbott B et al (LIGO Scientific Collaboration) 2007 Phys. Rev. D 76022001 (Preprint gr-qc/0703068)

[15] Golub G H and Van Loan C F 1989 Matrix Computations (Baltimore, MD: Johns Hopkins University Press)

[16] http://wwwcascina.virgo.infn.it/DataAnalysis/Noise/nap_index.html

[17] http://www.lsc-group.phys.uwm.edu/daswg/projects/matapps.html

[18] Whelan J T et al 2005 Class. Quantum Grav. 22 S1087 (Preprint gr-qc/0506025) 